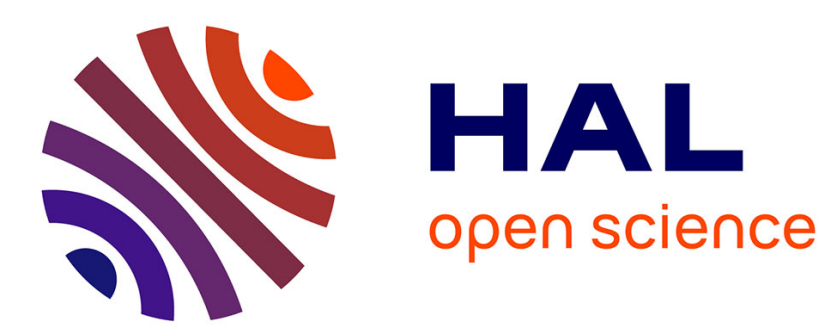

\title{
Constructive links between some morphological hierarchies on edge-weighted graphs
}

\author{
Jean Cousty, Laurent Najman, Benjamin Perret
}

\section{To cite this version:}

Jean Cousty, Laurent Najman, Benjamin Perret. Constructive links between some morphological hierarchies on edge-weighted graphs. International Symposium on Mathematical Morphology, May 2013, Uppsala, Sweden. pp.85-96. hal-00798622v2

\section{HAL Id: hal-00798622 \\ https://hal.science/hal-00798622v2}

Submitted on 2 Apr 2013

HAL is a multi-disciplinary open access archive for the deposit and dissemination of scientific research documents, whether they are published or not. The documents may come from teaching and research institutions in France or abroad, or from public or private research centers.
L'archive ouverte pluridisciplinaire HAL, est destinée au dépôt et à la diffusion de documents scientifiques de niveau recherche, publiés ou non, émanant des établissements d'enseignement et de recherche français ou étrangers, des laboratoires publics ou privés. 


\title{
Constructive links between some morphological hierarchies on edge-weighted graphs ${ }^{\star}$
}

\author{
Jean Cousty, Laurent Najman, and Benjamin Perret \\ Université Paris-Est, Laboratoire d'Informatique Gaspard-Monge, A3SI, ESIEE \\ $\{j$.cousty, l.najman, b. perret\}@esiee.fr
}

\begin{abstract}
In edge-weighted graphs, we provide a unified presentation of a family of popular morphological hierarchies such as component trees, quasi flat zones, binary partition trees, and hierarchical watersheds. For any hierarchy of this family, we show if (and how) it can be obtained from any other element of the family. In this sense, the main contribution of this paper is the study of all constructive links between these hierarchies.
\end{abstract}

\section{Introduction}

In recent years, (supervised) image segmentations in edge weighted graphs received a lot of attention. In this framework, several methods [1-5] were designed to segment images into partitions made of connected regions that are optimal in the sense of some well-known problems of combinatorial optimization such as min-cuts, random walks, or minimum spanning trees.

Some of these methods (see $[6,1]$ ) also satisfy a "scale consistency property" that assesses the robustness of the detected contours and regions over scales. Given three image seed points $x, y$, and $z$ that mark three objects of interest, a segmentation $S$ into three regions obtained from the three seeds $x, y$ and $z$ (i.e., each region contains one seed) "is consistent" with a segmentation $S^{\prime}$ into two regions obtained from the two seeds $x$ and $y$ if when a pixel belongs to the region of a seed in $S$, then it necessarily belongs to the region of $S^{\prime}$ that contains this seed. More generally, a segmentation is called hierarchical if it defines segmentations at different detail levels such that the segmentations at coarser levels can be obtained from those at finer levels by simple merge operations.

In fact, hierarchical segmentation methods are not limited to edge-weighted graphs (see e.g., [7-10]). In particular, in mathematical morphology, component trees [11], quasi-flat zones [12, 13], binary partition trees [14] and watersheds [1517] are hierarchies at the basis of efficient segmentation and filtering methods.

In this paper, we study these morphological hierarchies defined from edgeweighted graphs, and we provide a unified presentation of this family. For any hierarchy of this family, we show if (and how) it can be obtained from any other element of the family. In this sense, the main contribution of this paper is

* This work received funding from the Agence Nationale de la Recherche, contract ANR-2010-BLAN-0205-03. 


\begin{tabular}{|c|c|c|c|c|c|c|c|}
\hline & $\mathcal{P H}(G)$ & $\mathcal{M H}(G)$ & $\mathcal{P H}(T)$ & $\mathcal{M H}(T)$ & $\mathcal{Q}$ & $\mathcal{B}_{\prec}$ & $\mathcal{H}_{S}$ \\
\hline $\mathcal{P H}(G)$ & $\Longleftrightarrow$ & $\Longleftrightarrow$ & $\Longrightarrow$ & $\Longrightarrow$ & $\Longrightarrow$ & $\times$ & $\times$ \\
\hline $\mathcal{M H}(G)$ & $\Longleftrightarrow$ & $\Longleftrightarrow$ & $\Longrightarrow$ & $\Longrightarrow$ & $\Longrightarrow$ & $\times$ & $\times$ \\
\hline $\mathcal{P H}(T)$ & $\Longleftrightarrow$ & $\Longleftrightarrow$ & $\Longleftrightarrow$ & $\Longleftrightarrow$ & $\Longrightarrow$ & $\Longleftrightarrow$ & $\times$ \\
\hline $\mathcal{M H}(T)$ & $\Longleftrightarrow$ & $\Longleftrightarrow$ & $\Longleftrightarrow$ & $\Longleftrightarrow$ & $\Longrightarrow$ & $\Longleftrightarrow$ & $\times$ \\
\hline $\mathcal{Q}$ & $\Longleftrightarrow$ & $\Longleftrightarrow$ & $\Longleftrightarrow$ & $\Longleftrightarrow$ & $\Longleftrightarrow$ & $\Longrightarrow$ & $\times$ \\
\hline $\mathcal{B}_{\prec}$ & $\times$ & $\times$ & $\Longrightarrow$ & $\Longrightarrow$ & $\Longrightarrow$ & $\Longleftrightarrow$ & $\Longrightarrow$ \\
\hline $\mathcal{H}_{S}$ & $\times$ & $\times$ & $\times$ & $\times$ & $\times$ & $\Longleftrightarrow$ & $\Longleftrightarrow$ \\
\hline
\end{tabular}

Table 1. Summary of the main results. In the table, $T$ stands for any minimum spanning tree of $G, \mathcal{S}$ stands for any sequence of minima of $F, \mathcal{P H}(G)$ is the partitionhierarchy of $G, \mathcal{M H}(G)$ is the min-hierarchy of $G, \mathcal{P H}(T)$ is the partition-hierarchy of $T, \mathcal{M H}(T)$ is the min-hierarchy of $T, \mathcal{Q}$ is the quasi-flat zones hierarchy, $\mathcal{B}_{\prec}$ is the binary partition hierarchy by the ordering $\prec$, and $\mathcal{H}_{S}$ is an MSF hierarchy for $\mathcal{S}$. In a cell, the symbol $\Longleftarrow$ (resp. $\Longrightarrow$ ) indicates that the hierarchy corresponding to the column (resp. line) of the cell can be obtained from the one corresponding to the line (resp. column) of the cell, and the symbol $\Longleftrightarrow$ (resp. $\times$ ) indicates that two hierarchies can be (resp. cannot be) obtained one from each other.

the study of all constructive links between these morphological hierarchies. For establishing these links, the minimum spanning trees play a central role. Table 1 indicates all links that are shown in this paper. An important consequence of our results is the design of efficient algorithms based on Kruskal minimum spanning tree algorithm to compute these morphological hierarchies in quasi linear-time. These algorithms are presented in [18].

\section{Graphs}

We define a graph as a pair $X=(V(X), E(X))$ where $V(X)$ is a finite set and $E(X)$ is composed of unordered pairs of distinct elements in $V(X)$, i.e., $E(X)$ is a subset of $\{\{x, y\} \subseteq V(X) \mid x \neq y\}$. Each element of $V(X)$ is called a vertex or a point (of $G$ ), and each element of $E(X)$ is called an edge (of $X$ ).

Let $X$ and $Y$ be two graphs. If $V(X) \subseteq V(Y)$ and $E(X) \subseteq E(Y)$, then $X$ and $Y$ are ordered and we write $X \sqsubseteq Y$. If $X \sqsubseteq Y$, we say that $X$ is a subgraph of $Y$, or that $X$ is smaller than $Y$ and that $Y$ is greater than $X$. The intersection of $X$ and $Y$ is the graph $X \sqcap Y=\{V(X) \cap V(Y), E(X) \cap E(Y)\}$ and the union of $X$ and $Y$ is the graph $X \sqcup Y=\{V(X) \cup V(Y), E(X) \cup E(Y)\}$. The set of all subgraphs of a graph $G$ is denoted by $2^{G}$. The set $2^{G}$ equipped with the order relation $\sqsubseteq$ is a lattice whose infimum and supremum are the binary operations $\square$ and $\sqcup$ respectively (see [19] for a morphological study of this lattice).

Let $X$ be a graph. A path (in $X$ ) is a sequence $\left(x_{1}, \ldots, x_{n}\right)$ of points of $V(X)$ such that $\left\{x_{i}, x_{i+1}\right\} \in E$ for any $i$ in $[1, n-1]$. A path with no repeated vertex is said to be simple. The graph $X$ is connected if there exists a path between any two vertices of $X$. A (connected) component of $X$ is a subgraph $Y$ of $X$ that is connected and such that, for any connected graph $Z$, we have $Y=Z$ whenever the relation $Y \sqsubseteq Z \sqsubseteq X$ holds true. We denote by $\mathcal{C C}(X)$ the set of 
all components of $X$ and, if $x$ is a vertex in $V(X)$, we denote by $\mathcal{C C}_{x}(X)$ the unique element of $\mathcal{C C}(X)$ whose vertex set contains $x$.

Important notations. In the sequel of this paper, the symbol $G$ denotes a connected graph. Furthermore, to shorten the notations, its vertex and edge sets are denoted by $V$ and $E$ respectively instead of $V(G)$ and $E(G)$.

We finish this section with the presentation of an adjunction that is known for playing the role of a building block for morphology on graphs [19]. It will be useful for expressing several properties in the sequel of this article. We denote by $\epsilon$ the operator that maps to any subset $X$ of $V$ the subset of $E$ made of the edges of $G$ composed of two points in $X$, i.e., $\epsilon(X)=\{\{x, y\} \in E \mid x \in X, y \in X\}$. We denote by $\delta$ the operator that maps to any subset $X$ of $E$ the subset of $V$ that contains every vertex in $V$ which belongs to an edge in $X$, i.e., $\delta(X)=$ $\cup\{\{x, y\} \in X\}$. The pair $(\epsilon, \delta)$ is an adjunction [19]. Let $V^{\prime} \subseteq V$ and $E^{\prime} \subseteq E$. Using usual graph terminology, the graphs $\left(V^{\prime}, \epsilon\left(V^{\prime}\right)\right)$ and $\left(\delta\left(E^{\prime}\right), E\right)$ are called the graph induced by $V^{\prime}$ and the graph induced by $E^{\prime}$ respectively.

\section{$2 \quad$ Partitions and hierarchies}

For segmentation purposes, one is often interested in finding partitions of $V$. We denote by $2^{V}$ the set of all subsets of $V$. Recall that a subset $\mathcal{V}$ of $2^{V}$ whose elements are disjoint and nonempty is a partial partition (of $V$ ). The union of a partial partition is called its support. A partition (of $V$ ) is a partial partition whose support is $V$.

In the following, subgraphs of $G$ will be used to obtain partitions of $V$. Let $X$ be a subgraph of $G$. We denote by $\mathcal{V}_{C C}(X)$ the set that contains the vertex set of every component of $X$, i.e., $\mathcal{V}_{C C}(X)=\{V(Y) \mid Y \in \mathcal{C C}(X)\}$. Remark that the set $\mathcal{V}_{C C}(X)$ is a partial partition of $V$ whose support is $V(X)$. This partial partition is called the (partial) partition induced by $X$.

A set $\mathcal{H} \subseteq 2^{V}$ (resp. $\mathcal{H} \subseteq 2^{G}$ ) is a hierarchy on $V$ (resp. $G$ ) if any two elements of $\mathcal{H}$ are either disjoint or nested, i.e., for any $H_{1}, H_{2} \in \mathcal{H}$, we have $H_{1} \cap H_{2} \in\left\{\emptyset, H_{1}, H_{2}\right\}$ (resp. $H_{1} \sqcap H_{2} \in\left\{(\emptyset, \emptyset), H_{1}, H_{2}\right\}$ ). A hierarchy $\mathcal{H}$ on $V$ (resp. $G$ ) is complete if $V$ (resp. $G$ ) is in $\mathcal{H}$ and if for any $v \in V$, we have $\{v\} \in \mathcal{H},($ resp. $\{(\{v\}, \emptyset)\} \in \mathcal{H})$. It is well-known that the Hasse diagram of a hierarchy (resp. complete hierarchy) is a directed forest (resp. tree), often called the dendrogram of the hierarchy. In practice, this dendrogram is used as a representation of the hierarchy. Let $X$ and $Y$ be two distinct elements of a hierarchy $\mathcal{H}$ (on $V$ or $G$ ), following the terminology of the dendrogram, we say that $Y$ is a child of $X$ if $Y$ is the largest proper subset of $X$ among the elements of $\mathcal{H}$, i.e., if $Y \subseteq X$, and, for any $Z \in \mathcal{H}$ such that $Y \subseteq Z \subseteq X$, we have $Z=X$ or $Z=Y$. If $Y$ is a child of $X$, we say that $X$ is the parent of $Y$.

Let $\mathcal{H}$ be a hierarchy on $V$ (resp. $G$ ) and let $X$ be an element of $\mathcal{H}$. A minimum of $\mathcal{H}$ is an element of $\mathcal{H}$ that has no child. Let $\mathcal{C} \subseteq \mathcal{H}$. We say that $\mathcal{C}$ is a cut of $\mathcal{H}$ if i) the elements of $\mathcal{C}$ are pairwise disjoint, and ii) for any minimum $M$ of $\mathcal{H}$, the set $\mathcal{C}$ contains an element that is greater than $M$. If $\mathcal{H}$ is a hierarchy on $V$, we say that $\mathcal{H}$ is a hierarchy of partitions (on $V$ ) whenever any cut of $\mathcal{H}$ is a 
partition of $V$. The following property characterizes the hierarchies of partitions from their minima.

Property 1 Let $\mathcal{H}$ be a hierarchy on $V$. The hierarchy $\mathcal{H}$ is a hierarchy of partitions if and only if the set of its minima is a partition.

A direct corollary is that any complete hierarchy on $V$ is a hierarchy of partitions.

The hierarchies on $G$ may be used to obtain hierarchies (of partitions) on $V$. Let $\mathcal{H}$ be a hierarchy on $G$. We denote by $\mathcal{V}(\mathcal{H})$ the hierarchy on $V$ defined by $\mathcal{V}(\mathcal{H})=\{V(X) \mid X \in \mathcal{H}\}$ and we say that $\mathcal{V}(\mathcal{H})$ is the hierarchy (on $V$ ) induced by $\mathcal{H}$. Observe that the hierarchy $\mathcal{H}$ on $G$ induces a hierarchy of partitions on $V$ if and only if any vertex of $G$ is a vertex of a minimum of $\mathcal{H}$.

Let $\mathcal{H}$ be a hierarchy on $V$ (resp. on $G$ ), and let $x$ be in $V$. The greatest element of $\mathcal{H}$ that contains $x$ (resp. whose vertex set contains $x$ ) is denoted by $\mathcal{C C}_{x}(\mathcal{H})$. Observe that if $\mathcal{H}$ is complete, then $\mathcal{C C}_{x}(\mathcal{H})$ is exactly $V$ (resp. $G$ ).

\section{Component trees}

Intuitively, component trees [11] may be seen as hierarchies obtained from the connected components of an image. In particular, the min-tree is a well known hierarchical representation that is useful for anti-extensive connected operators. In this expression, the term min is used in reference to the leaves of these trees that are the regional minima of the images. In this section, we provide definitions of regional minima and of min-trees for edge-weighted graphs. Furthermore, on the same basis, we provide a definition of a hierarchy of partitions that allows links to be drawn between min-trees and quasi-flat zones.

Important notation. In the sequel, we denote by $F$ a function from $E$ to $\mathbb{R}^{+}$that weights the edges of $E$. Therefore, the pair $(G, F)$ is called an edgeweighted graph, and, for any $u \in E$, the value $F(u)$ is called the weight of $u$.

Let $k \in \mathbb{R}$. A subgraph $X$ of $G$ is a minimum of $F$ (at weight $k$ ) if i) $X$ is connected; and ii) $k$ is the weight of any edge of $X$; and iii) the weight of any edge adjacent to $X$ (i.e., any edge that contains exactly one vertex of $X$ ) is strictly greater than $k$.

In order to define the components of a weight map, the simple thresholding operation is used to produce level sets from which connected components can be considered. For given $\lambda \in \mathbb{R}$ and $X \subseteq E$, the $\lambda$-level set of $X$ (for $F$ ) is the set $\chi_{\lambda}(X)$ of all edges in $X$ whose value is not greater than $\lambda$, i.e., $\chi_{\lambda}(X)=$ $\{e \in X \mid F(e) \leq \lambda\}$. From the level set $\chi_{\lambda}(E)$ of $E$, two interesting graphs can be derived: the first one, called the $\lambda$-level graph of $G$, and denoted by $\chi_{\lambda}^{E}(G)$, is defined by $\chi_{\lambda}^{E}(G)=\left(\delta\left(\chi_{\lambda}(E)\right), \chi_{\lambda}(E)\right)$, and the second one, called the $\lambda$-level spanning graph of $G$ and denoted by $\chi_{\lambda}^{V}(G)$, is defined by $\chi_{\lambda}^{V}(G)=\left(V, \chi_{\lambda}(E)\right)$. More generally, if $X \sqsubseteq G$, the $\lambda$-level graph of $X$ and the $\lambda$-level spanning graph of $X$ are defined by $\chi_{\lambda}^{E}(X)=\left(\delta\left(\chi_{\lambda}(E(X))\right), \chi_{\lambda}(E(X))\right)$, and $\chi_{\lambda}^{V}(X)=$ $\left(V, \chi_{\lambda}(E(X))\right)$ respectively.

Note that $\chi_{\lambda}^{E}(G)$ can be derived from $\chi_{\lambda}^{V}(G)$ by removing all isolated points of $\chi_{\lambda}^{V}(G)$, and that, conversely, $\chi_{\lambda}^{V}(G)$ can be derived from $\chi_{\lambda}^{E}(G)$ by adding all 


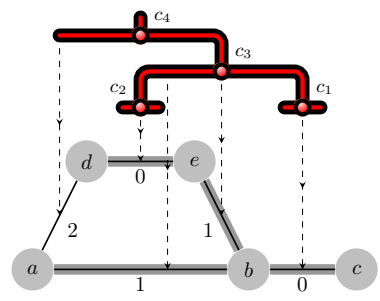

(a)

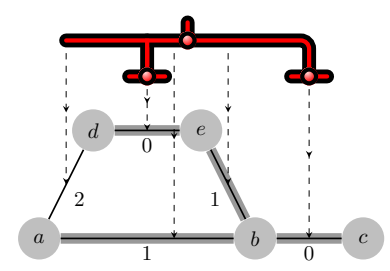

(b)

Fig. 1. Illustration of the min-hierarchy of a graph (a) and of its unique minimum spanning tree (b), which is represented by wide edges.

elements of $V$ to the vertex set of $\chi_{\lambda}^{E}(G)$. Hence, we always have $\chi_{\lambda}^{E}(G) \sqsubseteq \chi_{\lambda}^{V}(G)$. Note also that the partial partition induced by $\chi_{\lambda}^{V}(G)$ is always a partition of $V$ whereas the one induced by $\chi_{\lambda}^{E}(G)$ is in general not a partition (i.e., its support is in general a proper subset of $V$ ).

Definition 2 Let $X \sqsubseteq G$. The partition-hierarchy of $X$ (for $F$ ), denoted by $\mathcal{P H}(X)$, is the set $\mathcal{P H}(X)=\cup\left\{\mathcal{C C}\left(\chi_{\lambda}^{V}(X)\right) \mid \lambda \in \mathbb{R}\right\}$ and the min-hierarchy of $X$ (for $F$ ), denoted by $\mathcal{M H}(X)$, is the set $\mathcal{M H}(X)=\cup\left\{\mathcal{C C}\left(\chi_{\lambda}^{E}(X)\right) \mid \lambda \in \mathbb{R}\right\}$.

The Hasse diagram of the min-hierarchy of $G$ is known as the min-tree of $(G, F)$. Fig. 1a shows in red the min-tree of the edge-weighted graph represented in gray. The elements $C_{1}, C_{2}, C_{3}$ and $C_{4}$ of this min-hierarchy (i.e., the nodes of the min-tree) are represented by red horizontal lines. Observe that $C_{1}$ and $C_{2}$, which are the two components of the 0-level graphs of $G$, are the graphs induced by $\{\{b, c\}\}$ and $\{\{d, e\}\}$ respectively. The component $C_{3}$ (resp. $C_{4}$ ) is the unique connected component of the 1-level graph (resp. 2-level graph) of $G ; C_{3}$ is the graph induced by $\{\{a, b\},\{b, c\},\{b, e\},\{d, e\}\}$ and $C_{4}$ is the graph $G$ itself. The partition-hierarchy of $G$ is a superset of this min-hierarchy, which furthermore contains any subgraph of $G$ made of a single vertex. More generally, as assessed by the following property, the min-hierarchy and the partition-hierarchy of $G$ can always be obtained one from each other. Therefore, the min-hierarchy and the partition-hierarchy of $G$ are equivalent as well as the min-tree of $(G, F)$ and the Hasse diagram of the partition-hierarchy of $G$.

Property 3 The min-hierarchy of any subgraph $X$ of $G$ can be obtained by removing from the partition-hierarchy of $X$ the graphs made of a single vertex, i.e., $\mathcal{M H}(X)=\mathcal{P H}(X) \backslash\{(\{x\}, \emptyset) \mid x \in V\}$. Conversely, the partition-hierarchy of any subgraph $X$ of $G$ can be obtained by adding to the min-hierarchy of $X$ all graphs made of a single vertex, i.e., $\mathcal{P H}(X)=\mathcal{M H}(X) \cup\{(\{x\}, \emptyset) \mid x \in V\}$.

Observe that the partition-hierarchy of $G$ indeed induces a hierarchy of partitions on $V$, whereas, in general, the min-hierarchy of $G$ does not. In the next section, we will study the minimum spanning trees of $G$, and we will see that 
these particular subgraphs of $G$ are sufficient to recover the hierarchies of partitions induced by the min-hierarchy and the partition-hierarchy of $G$.

\section{Minimum spanning trees}

The minimum spanning tree is a typical and well-known problem of combinatorial optimization. It has been applied for many years to image analysis problems. The main result of this section states that the hierarchy of partitions induced by the partition-hierarchy of any minimum spanning tree of $G$ is exactly the same as the hierarchy of partitions induced by the partition-hierarchy of the graph $G$ itself. Furthermore, the minimum spanning trees are minimal (with respect to the relation $\sqsubseteq)$ for this property.

A graph $X$ is spanning (for $G$ ) if $V(X)=V$. Let $X \sqsubseteq G$. The weight of $X$ (for $F$ ), denoted by $F(X)$, is the sum of the weights of the edges in $E(X): F(X)=$ $\sum_{u \in E(X)} F(u)$. A connected spanning graph $T$ is a minimum spanning tree (of $(G, F)$ if the weight of $T$ is less than or equal to the weight of any other connected graph that is spanning.

Property 4 Let $T$ be any minimum spanning tree of $G$. Then, the partitions induced by $\chi_{\lambda}^{V}(T)$ and by $\chi_{\lambda}^{V}(G)$ are the same.

Let $X \sqsubseteq G$. We denote by $\phi(X)$ the graph induced by the vertex set of $X: \phi(X)=(V(X), \epsilon(V(X)))$. Note that $\phi$ is both a dilation and a closing in the lattice $2^{G}$ of all subgraphs of $G$ (for more details, see [19] where $\phi$ is denoted by $\alpha_{2}$ ). For a given hierarchy $\mathcal{H}$ of graphs, we write $\varphi(\mathcal{H})=\{\phi(X) \mid X \in \mathcal{H}\}$. It can be seen that $V(\phi(X))=V(X)$. Thus the hierarchies on $V$ induced by $\mathcal{H}$ and $\varphi(\mathcal{H})$ are the same, i.e., we always have $\mathcal{V}(\varphi(\mathcal{H}))=\mathcal{V}(\mathcal{H})$.

Given two hierarchies $\mathcal{H}_{1}$ and $\mathcal{H}_{2}$ whose elements are ordered by the relations $\leq_{1}$ and $\leq_{2}$ respectively, an (order) isomorphism from $\mathcal{H}_{1}$ to $\mathcal{H}_{2}$ is a bijection $f$ from $\mathcal{H}_{1}$ to $\mathcal{H}_{2}$ such that for any $X, Y \in \mathcal{H}_{1}, X \leq_{1} Y$ if and only if $f(X) \leq_{2} f(Y)$. If there exists an isomorphism from $\mathcal{H}_{1}$ to $\mathcal{H}_{2}$, then $\mathcal{H}_{1}$ and $\mathcal{H}_{2}$ are said isomorphic and we write $\mathcal{H}_{1} \cong \mathcal{H}_{2}$. Note that two hierarchies that are isomorphic can be represented by the same Hasse diagram.

Property 5 Let $T$ be any minimum spanning tree of $G$. Then the two following statements hold true:

1. $\mathcal{P H}(T) \cong \varphi(\mathcal{P H}(G))$; and

2. $\mathcal{V}(\mathcal{P H}(T))=\mathcal{V}(\varphi(\mathcal{P H}(G)))=\mathcal{V}(\mathcal{P H}(G))$.

In other words, the hierarchies induced by the partition-hierarchy of a minimum spanning tree and by the graph itself are the same. Furthermore, due to the mapping $\varphi$, the partition-hierarchy of any minimum spanning tree of $G$ can be recovered from the partition hierarchy of $G$. On the contrary, the converse is in general not true. Hence, in general, there is more information in the partitionhierarchy of $G$ than in partition-hierarchy of any of its minimum spanning trees. When available, such information may be used for further processing. 
Property 5 is illustrated on the edge-weighted graph $(G, F)$ of Fig. 1, where the red trees in (a) and (b) represent respectively $\mathcal{P H}(G)$ and $\mathcal{P H}(T) \cong \varphi(\mathcal{P H}(G))$, $T$ being the minimum spanning tree depicted with "wide" edges.

\section{Quasi-flat zones}

The quasi-flat zones (see e.g. $[12,13,8]$ ) have been studied since the 70 's, and they have been used recently as a basis for constrained connectivity segmentations. In this section, we investigate the links between the quasi-flat zones, the min-trees, the partition-hierarchies of a graph $G$ and of its minimum spanning trees.

Let $\lambda \in \mathbb{R}$. A path $\pi=\left(x_{0}, \ldots, x_{n}\right)$ is $\lambda$-connected if for any $i$ in $\llbracket 0, n-1 \rrbracket=$ $\{0, \ldots n-1\}$, we have $\left\{x_{i}, x_{i+1}\right\} \in E$ and $F\left(\left\{x_{i}, x_{i+1}\right\}\right) \leq \lambda$. For any two vertices $x$ and $y$ in $V$, we set $\lambda-\Pi(x, y)$ as the set of all $\lambda$-connected paths from $x$ to $y$. The $\lambda$-flat zone (or quasi-flat zone at level $\lambda$ ) of a vertex $x$ is the set $\lambda-C C(x)=\cup\{y \in V \mid \lambda-\Pi(x, y) \neq \emptyset\}$. The set $Q_{\lambda}=\{\lambda-C C(x) \mid x \in V\}$ of $\lambda$-flat zones over all vertices in $E$ is a partition.

Definition 6 The set $\mathcal{Q}=\cup\left\{Q_{\lambda} \mid \lambda \in \mathbb{R}\right\}$ is the quasi-flat zones hierarchy of $F$.

The quasi-flat zones hierarchy is a complete hierarchy, and thus also a hierarchy of partitions. In the literature, the term $\alpha$-tree was coined by G. Ouzounis and P. Soille for the Hasse diagram of the quasi-flat zones hierarchy [20].

For any $\lambda \in \mathbb{R}$, it can be seen that $Q_{\lambda}$ is the partition induced by the $\lambda$-level graph $\chi_{\lambda}^{V}(G)$ of $G$. Hence, by Property 4 , the partition $Q_{\lambda}$ is also the partition induced by the $\lambda$-level graph of any minimum spanning tree of $G$. Therefore, the following property linking the quasi-flat zones hierarchy to the partitionhierarchies of any minimum spanning tree of $G$ can be established.

Property 7 Let $T$ be a minimum spanning tree of $G$. Then, the two following statements hold true:

1. $Q \cong \mathcal{P H}(T) ;$ and

2. $Q=\mathcal{V}(\mathcal{P H}(T))$.

The first relation states that the quasi-flat zones hierarchy and the partitionhierarchy of $T$ are isomorphic. Due to Property 5.1, we deduce that these two hierarchies are also isomorphic to $\varphi(\mathcal{P H}(G))$ obtained by simplifying the partitionhierarchy of $G$. Furthermore, by Property 3, we deduce that these two hierarchies may also be obtained form the min-hierarchy of $G$. Hence, Property 7.1 states that the $\alpha$-tree and the partition-tree of any minimum spanning tree of $G$ are the same and that they both can be obtained from the partition- and min-trees of the graph $G$ itself. The second relation states that the quasi-flat zones hierarchy is exactly the hierarchy of partitions induced by the partition-hierarchy of any minimum spanning tree of $G$. It thus states how to obtain the quasi flat zones hierarchy from any other hierarchy previously presented in this paper. 


\section{$6 \quad$ Binary partition trees}

In this section, we present the binary partition hierarchies by (altitude) orderings. These hierarchies fall into the wide category of binary partition trees as introduced by P. Salembier [21]. Then, we state that the quasi-flat zones hierarchy can be recovered from this hierarchy, and we show a mapping from the elements of these hierarchies to the edge-set of the minimum spanning trees of $(G, F)$. Note that Meyer studied similar links between catchment basins and minimum spanning trees in [17].

Let $\prec$ be a total ordering on $E$, i.e., $\prec$ is a binary relation that is transitive and trichotomous (for any $u$ and $v$ in $E$ only one of the relations $u \prec v, v \prec u$, and $u=v$ holds true). Let $k$ be any element in $\llbracket 1,|E| \rrbracket$, we denote by $u_{k}^{\prec}$ the $k$-th element of $E$ with respect to $\prec$.

Definition 8 Let $k$ be an element in $\llbracket 1,|E| \rrbracket$. We set $\mathcal{B}^{0}=\{\{x\} \mid x \in V\}$. The partial binary partition hierarchy $\mathcal{B}^{k}$ at rank $k$ (by the ordering $\prec$ ) is the hierarchy on $V$ defined by $\mathcal{B}^{k}=\mathcal{B}^{k-1} \cup\left\{\mathcal{C C}_{x}\left(\mathcal{B}^{k-1}\right) \cup \mathcal{C C}_{y}\left(\mathcal{B}^{k-1}\right)\right\}$ where $u_{k}^{\prec}=$ $\{x, y\}$.

The partial binary partition hierarchy at rank $|E|$ is called the binary partition hierarchy by (the ordering) $\prec$ and it is denoted by $\mathcal{B}_{\prec}$.

The Hasse diagram of the binary partition hierarchy is known in the literature as the binary partition tree (see, e.g., Fig. 2a). Note that, for every possible value of $k$, the partial binary partition hierarchy at rank $k$ is a hierarchy of partitions and furthermore the binary partition hierarchy is a complete hierarchy.

Let $\prec$ be an ordering on $E$, and let $k \in \llbracket 1,|E| \rrbracket$. Observe that the partial binary partition hierarchy at rank $k$ is equal to the partial binary partition hierarchy at rank $k-1$ if and only if the $k$-th edge for $\prec$ links two vertices that are already in a same set of $\mathcal{B}^{k-1}$ (see e.g. the hierarchies $\mathcal{B}^{6}$ and $\mathcal{B}^{7}$ in Fig. 2a). Hence, we may associate to any element $X$ in $\mathcal{B}_{\prec}^{\star}=\mathcal{B}_{\prec} \backslash \mathcal{B}^{0}$ the lowest rank at which a partial binary partition tree contains $X$. This rank is called the rank of $X$, it is denoted by $r(X)$ and we have $r(X)=\min \left\{k \in \llbracket 1, n \rrbracket \mid X \in \mathcal{B}^{k}\right\}$. This rank also allows us to directly map the elements of $\mathcal{B}^{\star}$ to a subset of $E$. Let $X \in \mathcal{B}_{\prec}^{\star}$, the building edge of $X$ is the $r(X)$-th edge of $E$ for $\prec$. The set of building edges of all elements in $\mathcal{B}_{\prec}^{\star}$ is called the building set of $\mathcal{B}_{\prec}$.

We say that an ordering $\prec$ on $E$ is an altitude ordering (for $F$ ) if $F(u) \leq F(v)$ for any two $u$ and $v$ in $E$ such that $u \prec v$. If there is only one altitude ordering for $F$, then we say that $F$ is totally ordering.

Property 9 Let $\prec$ be an altitude ordering, and let $\mathcal{B}_{\prec}$ be the binary partition hierarchy by $\prec$. If $F$ is totally ordering, then the two following statements hold true:

1. the graph induced by the building set of $\mathcal{B}_{\prec}$ is the unique minimum spanning tree of $F$; and

2. $\mathcal{B}_{\prec}=\mathcal{Q}$. 


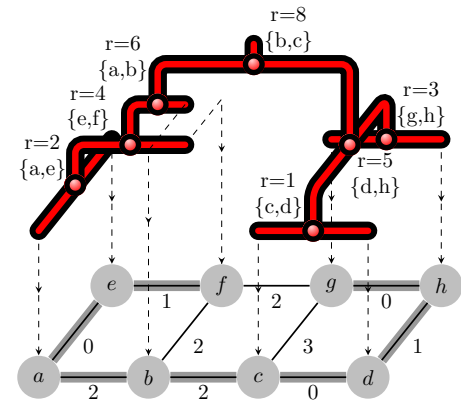

(a)

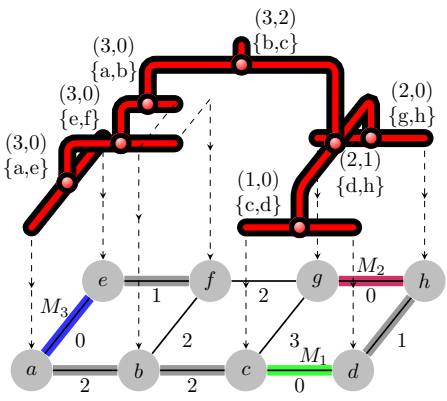

(c)

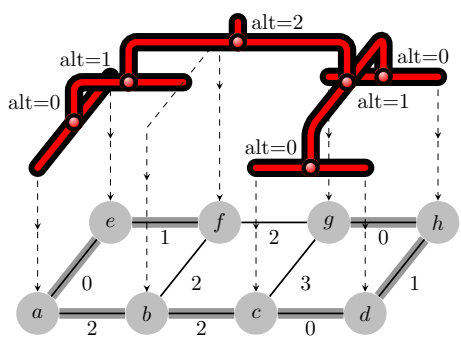

(b)

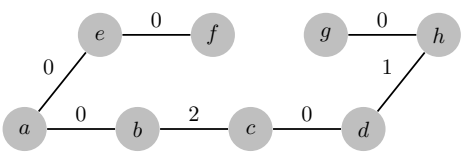

(b)

Fig. 2. a: A binary partition hierarchy/tree $\mathcal{B}_{\prec}$ for the altitude ordering $\{c, d\} \prec$ $\{a, e\} \prec\{g, h\} \prec\{e, f\} \prec\{d, h\} \prec\{a, b\} \prec\{b, f\} \prec\{b, c\} \prec\{f, g\} \prec\{c, g\}$. b: $\Psi\left(\mathcal{B}_{\prec}\right)$. c: The same binary partition hierarchy with a sequence $\mathcal{S}=\left\langle M_{1}, M_{2}, M_{3}\right\rangle$ of minima of $F$ and, for each non-leaf element, a pair of values (ext,pers) made of the extinction of the component and the persistence of its building edge (note that the extinction of a leaf/singleton is always 0 ). $\mathrm{d}$ : The edge-weighted graph whose partition-hierarchy is the hierarchy induced by $\prec$ and $\mathcal{S}$, which is thus also the MSF hierarchy for $\mathcal{S}$.

Hence, under the conditions of the previous property, we deduce from Properties 7 and 5 that $\mathcal{B}_{\prec}$ is also isomorphic to $\mathcal{P H}(T)$ and to $\varphi(\mathcal{P H}(G))$. Thus, it can be obtained as a simplification of the partition-hierarchy $\mathcal{P H}(G)$ and also, by Property 3 , as a simplification of the min-hierarchy $\mathcal{M H}(G)$.

Let $\prec$ be an altitude ordering. Let $X \in \mathcal{B}_{\prec}^{\star}$, we call altitude of $X$, the weight of its building edge. We say that $X$ is principal for $\mathcal{B}_{\prec}$ if it has no parent or if its altitude is less than the one of its parent. The set of all principal elements of $\mathcal{B}_{\prec}$ is denoted $\Psi\left(\mathcal{B}_{\prec}\right)$ (see Figs. 2a and b for illustrations).

Property 10 Let $\prec$ be an altitude ordering, and let $\mathcal{B}_{\prec}$ be the binary partition hierarchy by $\prec$. Then, the two following statements hold true:

1. the graph induced by the building set of $\mathcal{B}_{\prec}$ is a minimum spanning tree of $F$; and 
2. $\mathcal{Q}=\Psi\left(\mathcal{B}_{\prec}\right)$.

The previous property states that the quasi-flat zones hierarchy $\mathcal{Q}$ can be obtained by simplifying the binary partition hierarchy $\mathcal{B}_{\prec}$. In fact, contrarily to the case of maps which are totally ordering, the converse is, in general, not true: the binary partition hierarchy cannot be obtained from the quasi-flat zones hierarchy or from the partition-hierarchy of a minimum spanning tree. Furthermore, it can be shown that, in general, one cannot recover a binary partition hierarchy from a min-/partition-hierarchy of $G$ either.

\section{$7 \quad$ Hierarchies of minimum spanning forests}

This section first presents the minimum spanning forests rooted in subgraphs of $G$. This notion of a forest, which is useful for (seeded) image segmentation, is known to be equivalent to the one of minimum spanning tree. Then, hierarchies of minimum spanning forests are introduced. Each such hierarchy induces a hierarchy of partitions on $V$. Finally, we state the main result of this section that shows how hierarchies of minimum spanning forests can be obtained from binary partition hierarchies.

Let $X$ and $Y$ be two nonempty subgraphs of $G$. We say that $Y$ is rooted in $X$ if $V(X) \subseteq V(Y)$ and if the vertex set of any component of $Y$ contains the vertex set of exactly one component of $X$. We say that $Y$ is a minimum spanning forest (MSF) rooted in $X$ (with respect to $F$ ) if i) $Y$ is spanning; ii) $Y$ is rooted in $X$; and iii) the weight of $Y$ is less than or equal to the weight of any graph $Z$ satisfying (1) and (2) (i.e., $Z$ is both spanning and rooted in $X$ ). Furthermore, any minimum spanning tree of $G$ is called an MSF rooted in the empty graph.

For instance, the graphs induced by the null edges of Fig. 2d is an MSF rooted in the graph made of the minima $M_{1}, M_{2}$ and $M_{3}$ shown in Fig. 2c.

A possible definition for watershed, called watershed-cuts, follows the drop of water principle. In [4], we have proved the equivalence between MSF rooted in the set of minima and watershed cuts. In practice, watersheds from markers are often computed, and subsets of minima of the original edge-weighted graph constitute robust markers. The next definition presents a notion of hierarchy of MSFs rooted in such subsets.

We denote by $\mathcal{M}_{F}$ the set of all minima of $F$.

Definition 11 (MSF hierarchy, [6]) Let $\mathcal{S}=\left\langle M_{1}, \ldots, M_{\ell}\right\rangle$ be a sequence of pairwise distinct minima of $F$ and let $\left\langle X_{0}, \ldots, X_{\ell}\right\rangle$ be a sequence of subgraphs of $G$ such that:

1. for any $i \in \llbracket 0, \ell \rrbracket, X_{i}$ is an $M S F$ rooted in $\sqcup\left[\mathcal{M}_{F} \backslash\left\{M_{j} \mid j \in \llbracket 1, i \rrbracket\right\}\right]$; and

2. for any $i \in \llbracket 1, \ell \rrbracket$, we have $X_{i-1} \sqsubseteq X_{i}$.

The set $\mathcal{T}=\cup\left\{C C\left(X_{i}\right) \mid i \in \llbracket 0, \ell \rrbracket\right\}$ is called an MSF hierarchy for $\mathcal{S}$. 
Let $\prec$ be an altitude ordering on $E$, let $\mathcal{S}=\left\langle M_{1}, \ldots, M_{\ell}\right\rangle$ be a sequence of pairwise distinct minima of $F$, and let $X \in \mathcal{B}_{\prec}$. The extinction value of $X$ for $\mathcal{S}$ is 0 if there is no element of $\mathcal{S}$ whose vertex set is included in $X$, or, otherwise, it is set to the highest index $k$ such that the vertex set of $M_{k}$ is included in $X$.

Intuitively, if we see the sequence $\mathcal{S}$ as a sequence of "markers" ranked by increasing "importance", the extinction value of a set $X$ in $\mathcal{H}$ can be seen as the rank of the most important marker of $X$ (i.e., that is contained in $X$ ). For instance, in Fig. 2, the extinction value of every component of the binary partition tree is given for the sequence $\left\langle M_{1}, M_{2}, M_{3}\right\rangle$, where $M_{1}$ (resp. $M_{2}$ and $M_{3}$ ) is the minimum induced by $\{\{c, d\}\}$ (resp. $\{\{g, h\}\}$ and $\{\{e, f\}\}$ ). Dually, one can intuitively consider the persistence of an edge $u$ as the highest rank $k$ such that the vertices linked by $u$ belong to distinct regions of the partitions obtained by considering only the $k$ most important markers (see e.g. Figs. 2c, and d). Based on this notion of persistence, Property 12 states that MSF hierarchies can be obtained using only the binary partition trees by altitude orderings.

Let $\prec$ be an altitude ordering on $E$. Let $\mathcal{S}=\left\langle M_{1}, \ldots, M_{\ell}\right\rangle$ be a sequence of pairwise distinct minima of $F$. Let $u$ be an edge in the building set of $\mathcal{B}_{\prec}$, and let $X$ be the unique element in $\mathcal{B}_{\prec}$ whose building edge is $u$. The persistence value of $u$ is the minimum of the extinction values of the children of $X$. Let $i \in \llbracket 1, \ell \rrbracket$. We denote by $B_{i}$ the set of building edges whose persistence value is lower than or equal to $i$ and the set of graphs $\cup\left\{\mathcal{C C}\left(\left(V, B_{i}\right)\right) \mid i \in \llbracket 1, \ell-1 \rrbracket\right\}$ is called the hierarchy induced by $\prec$ and $\mathcal{S}$.

Property 12 Let $\mathcal{S}=\left\langle M_{1}, \ldots, M_{\ell}\right\rangle$ be a sequence of pairwise distinct minima of $F$ and let $\mathcal{T}$ be a hierarchy on $G$. The hierarchy $\mathcal{T}$ is an MSF hierarchy for $\mathcal{S}$ if and only if there exists an altitude ordering $\prec$ such that $\mathcal{T}$ is the hierarchy induced by $\prec$ and $\mathcal{S}$.

\section{Conclusion}

This paper investigates the links between some popular morphological hierarchies. Table 1 sums up the links shown in this paper. These links open the way towards a family of efficient algorithms, based on Kruskal minimum spanning tree algorithms, for computing morphological hierarchies. These algorithms are presented in the companion paper [18]. Furthermore, the links established in this paper invites us to bridge hierarchical processing coming from different family of hierarchies. Evaluating the impact of mixing these techniques is left for future work. It also allows for designing new hierarchical methods derived from image predicate which are not necessarily hierarchical (see a first example in [22]). Finally, the links between the hierarchical methods presented in this paper and those based on self-dual tree of level lines [23] still need to be investigated. 


\section{References}

1. Morris, O.J., Lee, M.d.J., Constantinides, A.G.: Graph theory for image analysis: an approach based on the shortest spanning tree. IEE proc. on communications, radar and signal 133(2) (1986) 146-152

2. Boykov, Y., Veksler, O., Zabih, R.: Fast approximate energy minimization via graph cuts. PAMI 23(11) (2001) 1222-1239

3. Grady, L.: Random walks for image segmentation. PAMI 28(11) (2006) 1768-1783

4. Cousty, J., Bertrand, G., Najman, L., Couprie, M.: Watershed Cuts: Minimum Spanning Forests and the Drop of Water Principle. PAMI 31(8) (2009) 1362-1374

5. Couprie, C., Grady, L., Najman, L., Talbot, H.: Power Watersheds: A Unifying Graph Based Optimization Framework. PAMI 33(7) (2011) 1384-1399

6. Cousty, J., Najman, L.: Incremental algorithm for hierarchical minimum spanning forests and saliency of watershed cuts. In: ISMM. Volume 6671 of LNCS. (2011) $272-283$

7. Guigues, L., Cocquerez, J.P., Men, H.L.: Scale-sets image analysis. IJCV 68(3) (2006) 289-317

8. Soille, P.: Constrained connectivity for hierarchical image partitioning and simplification. PAMI 30(7) (2008) 1132-1145

9. Arbelaez, P., Maire, M., Fowlkes, C., Malik, J.: Contour detection and hierarchical image segmentation. PAMI 33(5) (2011) 898-916

10. Pont-Tuset, J., Marques, F.: Supervised assessment of segmentation hierarchies. In: Computer Vision - ECCV 2012. Volume 7575 of LNCS. (2012) 814-827

11. Salembier, P., Oliveras, A., Garrido, L.: Anti-extensive connected operators for image and sequence processing. TIP 7(4) (1998) 555-570

12. Nagao, M., Matsuyama, T., Ikeda, Y.: Region extraction and shape analysis in aerial photographs. CGIP 10(3) (1979) 195-223

13. Meyer, F., Maragos, P.: Morphological scale-space representation with levelings. In: Scale-Space Theories in Computer Vision. Volume 1682 of LNCS. (1999) 187-198

14. Salembier, P., Garrido, L.: Binary partition tree as an efficient representation for image processing, segmentation, and information retrieval. TIP 9(4) (April 2000) 561-576

15. Beucher, S.: Watershed, hierarchical segmentation and waterfall algorithm. In: ISMM. (1994) 69-76

16. Najman, L., Schmitt, M.: Geodesic saliency of watershed contours and hierarchical segmentation. PAMI 18(12) (1996) 1163-1173

17. Meyer, F.: The dynamics of minima and contours. In: ISMM. (1996) 329-336

18. Najman, L., Cousty, J., Perret, B.: Playing with kruskal: algorithms for morphological trees in edge-weighted graphs. In: ISMM. (2013) This volume.

19. Cousty, J., Najman, L., Dias, F., Serra, J.: Morphological filtering on graphs. CVIU 117 (2012) 370-385

20. Ouzounis, G., Soille, P.: Pattern spectra from partition pyramids and hierarchies. In: ISMM. Volume 6671 of LNCS. (2011) 108-119

21. Salembier, P., Garrido, L.: Binary partition tree as an efficient representation for image processing, segmentation and information retrieval. TIP 9(4) (2000) 561-576

22. Guimarães, S.J.F., Cousty, J., Kenmochi, Y., Najman, L.: A hierarchical image segmentation algorithm based on an observation scale. In: SSSPR. Volume 7626 of LNCS. (2012) 116-125

23. Caselles, V., Monasse, P.: Geometric Description of Images as Topographic Maps. Volume 1984 of LNCS. Springer (2010) 